\title{
Editorial
}

Notfall Rettungsmed 2019 $\cdot 22: 675-676$

https://doi.org/10.1007/s10049-019-00656-9

(c) Springer Medizin Verlag GmbH, ein Teil von Springer Nature 2019

\author{
Christian Both ${ }^{1}$ Florian Hoffmann ${ }^{2}$ \\ ${ }^{1}$ Kinderspital Zürich - Eleonorenstiftung, Anästhesieabteilung, Zürich, Schweiz \\ ${ }^{2}$ Kinderklinik und Kinderpoliklinik im Dr. von Haunerschen Kinderspital, Interdisziplinäre \\ Kinderintensivstation - Kindernotfallmedizin, Klinikum der Universität München, München, Deutschland
}

\section{Rubrik „Pädiatrische Notfälle“ neu aufgelegt}

\author{
Zusatzmaterial online \\ Die Online-Version dieses Beitrags (https:// \\ doi.org/10.1007/s10049-019-00656-9) \\ enthält eine Manuskriptvorlage für eigene \\ Fallberichte für die Rubrik. Sollten Sie diese \\ nicht runterladen können, wenden Sie sich \\ bitte an die Redaktion. \\ Beitrag und Zusatzmaterial stehen Ihnen auf \\ www.springermedizin.de zur Verfügung. \\ Bitte geben Sie dort den Beitragstitel in die \\ Suche ein, das Zusatzmaterial finden Sie beim \\ Beitrag unter "Ergänzende Inhalte".
}

Liebe Leserinnen und Leser,

präklinische Notfälle bei Kindern machen nur einen kleinen Prozentsatz der Einsätze des Rettungsdienstes aus. Gleichzeitig aber stellen diese alle Beteiligten immer wieder vor immense Herausforderungen. Leitlinien werden nicht eingehalten, es kommt zu folgenschweren Dosierungsfehlern bei der gewichtsadaptierten Applikation von Medikamenten, die unkoordinierte Zusammenarbeit im Team führt zu Kommunikationsproblemen und der Umgang mit den Eltern bereitet Schwierigkeiten. Diese Probleme spiegeln sich im Wunsch der Leserbefragung von Notfall+Rettungsmedizin wider, Kindernotfällen noch mehr Bedeutung beizumessen.

Die beiden pädiatrischen Urväter dieser Zeitschrift Prof. Dr. Thomas Nicolai aus München und Prof. Dr. Markus Weiss aus Zürich haben ihre langjährige Tätigkeit als Herausgeber der Rubrik „Pädiatrische Notfälle" beendet und an den Nachwuchs übergeben. Wir wollen beiden an dieser Stelle für ihr unermüdliches Engagement und die große Motivation danken. Beide haben sich für die Versor- gung kritisch kranker Kinder und die präklinische Notfallmedizin mehr wie verdient gemacht und somit vielen Erstversorgern und damit auch vielen Kindern geholfen.

Wir, Christian Both aus Zürich und Florian Hoffmann aus München, dürfen dieses Erbe nun antreten. Wir kommen beide aus dem Bereich der präklinischen Notfallmedizin und wollen auch in Zukunft dazu beitragen, dass sich die Leserinnen und Leser von Notfall+Rettungsmedizin gut im Bereich der Kindernotfälle fortgebildet fühlen.

Als Herausgeber der Rubrik „Pädiatrische Notfälle" wollen wir nun neben der Begutachtung von frei eingereichten Beiträgen auch aktiv Themen in die Zeitschrift bringen. Unser Anliegen ist es, in jeder zweiten Ausgabe ein wichtiges pädiatrisches Thema aus der Notfallversorgung anzubieten. Ziel ist eine praxisorientierte und pragmatische Berichterstattung zu den wichtigsten pädiatrischen Notfallsituationen von erfahrenen Kolleginnen und Kollegen.

Gleichzeitig wollen wir Sie als Leserinnen und Leser einladen, Kolleginnen und Kollegen an eigenen interessanten Fallberichten teilhaben zu lassen und uns ihre Fälle in zugrundeliegender Vorlage zukommen zu lassen. Einen Vorschlag für die Struktur und Gestaltung solcher Fallberichte finden Sie als Download in der online Version des Beitrags auf www. springermedizin.de (s. Infobox am Anfang des Beitrags). Alle Beiträge werden durch Experten begutachtet und in die aktuelle Studienlage eingeordnet.

Respiratorische Notfälle stellen eine der häufigsten Notfallsituationen im Kindesalter dar. Der erste Beitrag in dieser 


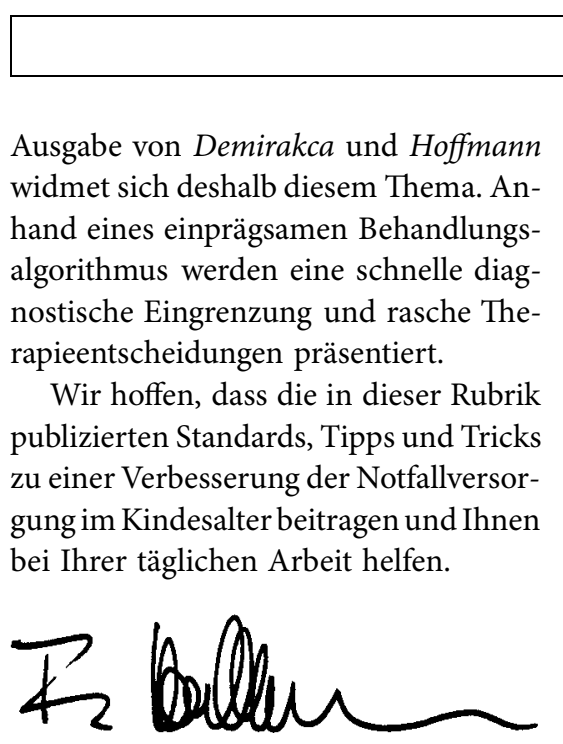

Dr. Christian Both, Zürich
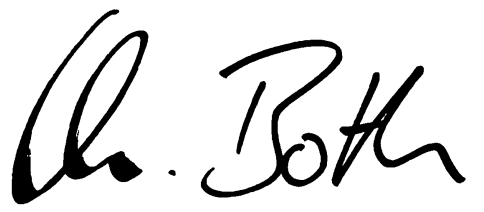

PD Dr. Florian Hoffmann, München

\section{Korrespondenzadresse}

Dr. Christian Both
Kinderspital Zürich
- Eleonorenstiftung,
Anästhesieabteilung
Steinwiesstrasse 75,
8032 Zürich, Schweiz
christian.both@kispi.uzh.ch

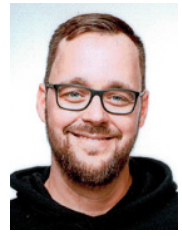

PD Dr. Florian Hoffmann Kinderklinik und Kinderpoliklinik im Dr. von Haunerschen Kinderspital, Interdisziplinäre Kinderintensivstation Kindernotfallmedizin, Klinikum der Universität München

Lindwurmstr. 4, 80337 München, Deutschland florian.hoffmann@med.unimuenchen.de

Interessenkonflikt. C. Both und F. Hoffmann geben an, dass kein Interessenkonflikt besteht.

\section{Fachnachrichten}

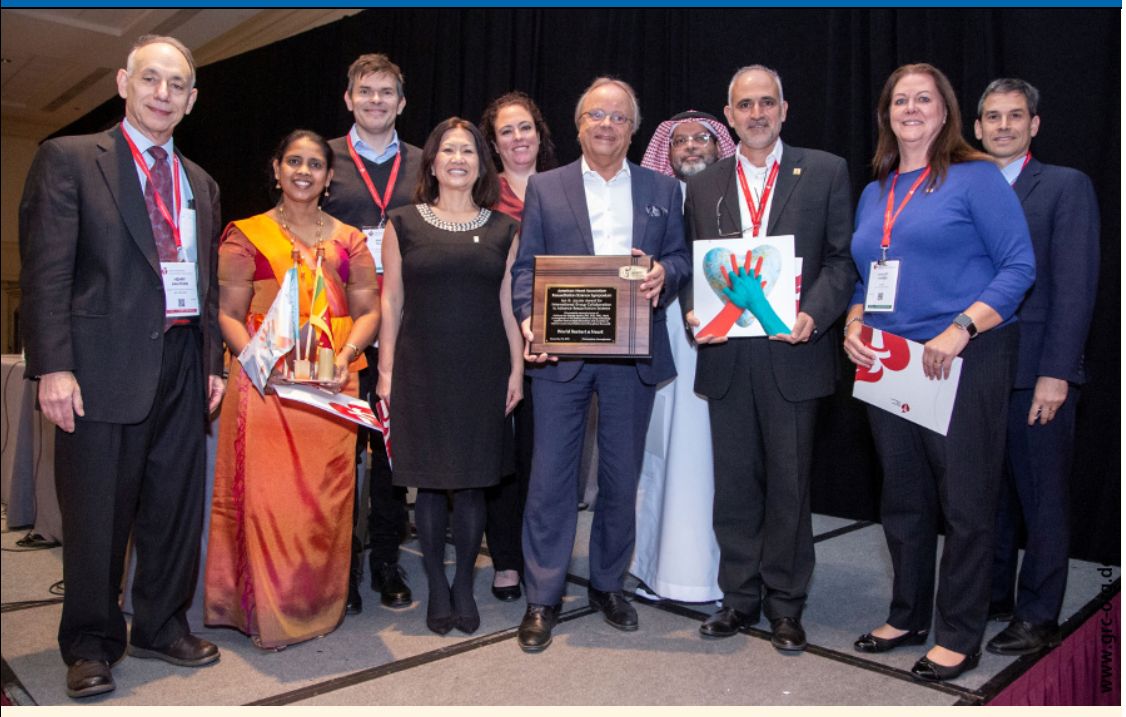

\section{Internationale Kooperation zur Reanimation geehrt}

Mit seiner gesamten weltweiten Arbeitsgruppe ist Univ.-Prof. Dr. Bernd Böttiger, Vorstandsvorsitzender des GRC und Direktor der Anästhesiologie und Operativen Intensivmedizin der Uniklinik Köln, beim Resuscitation Science Symposium der American Heart Association (AHA) Mitte November zum wiederholten Mal mit dem lan G. Jacobs Award ausgezeichnet worden. Der Award ehrt internationale Kollaborationen im Bereich der Reanimationswissenschaften. Die Gruppe erhielt die Auszeichnung für ihre Aktivitäten im Rahmen der "World Restart a Heart"Initiative.

Bereits im Frühjahr 2012 unterschrieben 400 Abgeordnete im Europaparlament auf Anregung des European Resuscitation Council (ERC) eine Deklaration zur Einführung des European Restart a Heart Day zur Steigerung der Laienreanimation. Der European Restart a Heart Day fand erstmals am 16. Oktober 2013 unter dem Motto „KIDS SAVE LIVES“ in mehr als 20 europäischen Ländern statt.

Mit Unterstützung des ERC und des Deutschen Rates für Wiederbelebung, dessen Vorstandsvorsitzender Univ.-Prof. Bernd W. Böttiger ist, wurde dann die weltweite „World Restart a Heart"Initiative unter dem Dach des International Liaison Committee on Resuscitation (ILCOR) initiiert. Seit 2018 wird nun jährlich an und rund um den 16. Oktober - oder in den Wochen davor und danach - zu Aktionen und Veranstaltungen zum Thema Laienreanimation aufgerufen. Das sind beispielsweise Wiederbelebungs-Massentrainings, Infokampagnen oder Auffrischungskurse, wie auch im vergangenen Monat am Kölner Dom. Primäres Ziel des Tages ist es, ein Bewusstsein für das Thema zu schaffen und weltweit möglichst viele Laien in Wiederbelebungsmaßnahmen zu schulen. Bereits der Auftakt im Jahr 2018 war sehr erfolgreich. Weltweit wurden in 2018 und 2019 über 1,5 Millionen Menschen in Reanimation trainiert, und über die sozialen Medien erreichte die Initiative fast 30 Millionen Menschen.

Der lan G. Jacobs Award wird seit 2010 an internationale Forschungsgruppen verliehen, die sich in besonderer Weise im Bereich der erweiterten Reanimationsversorgung engagieren und hierbei richtungsweisende Ergebnisse mit hoher Relevanz für die Versorgung von Menschen mit Herzkreislaufstillstand hervorbringen. Der Award wird von der American Heart Association (AHA) zu Ehren von Professor lan George Jacobs, PhD, OStJ, FERC, FAHA verliehen, der sich unermüdlich dafür eingesetzt hat, Mediziner und Wissenschaftler unterschiedlichster Disziplinen weltweit zusammenzubringen, um die globale Versorgung von Menschen mit plötzlichem Herzkreislaufstillstand zu verbessern. 